\title{
Estudio prospectivo y randomizado de suplementación de hierro temprana versus tardía en niños con peso al nacer menor de $1.301 \mathrm{~g}$
}

\author{
DIEGO SACAQUIRÍN W. ${ }^{1}$, HUGO SALVO F. ${ }^{1}$, RICARDO SALINAS G. ${ }^{2}$ \\ 1. Pediatra Neonatólogo, Hospital Luis Tisné B. \\ 2. Pediatra Neonatólogo, Facultad de medicina, Campus Norte Universidad de Chile.
}

\begin{abstract}
Prospective randomized study of early versus late enteral iron supplementation in children who weighed $<1,301$ grams at birth

Objective: Determine whether early iron supplementation would decrease the need, the number and volume of transfused red blood cells in relation to late iron supplementation in children with birth weight less than 1,301 g. Patients and Methods: Very low birth weight (VLBW) infants were randomly assigned to receive early iron supplementation of $3 \mathrm{mg} / \mathrm{kg} /$ day as soon as they could tolerate enteral feeding of $100 \mathrm{ml} / \mathrm{kg} / \mathrm{day}$, or at 61 days of life as late supplementation. Hemoglobin levels were measured at the beginning of early iron supplementation and at 2 months of age. The red blood cell transfusion was performed according to transfusion guidelines and erythropoietin was not administered. Results: No differences were observed regarding the number of red cell transfusions between the groups. Morbidities associated with prematurity presented no significant differences. Conclusions: Early iron supplementation to a child that is tolerating $100 \mathrm{~mL} / \mathrm{kg} / \mathrm{day}$ of milk, does not decrease the incidence of red blood cell transfusions compared to late start iron at 61 days of life, and it is probably safe in infants with birth weight $<1,301 \mathrm{~g}$.

(Key words: Anemia, prematurity, ferrous sulfate, transfusion, red blood cells).

Rev Chil Pediatr 2013; 84 (4): 379-386
\end{abstract}

\section{RESUMEN}

Objetivo: Determinar si la suplementación temprana de hierro disminuye la necesidad, el número y volúmen de glóbulos rojos transfundidos, en relación a la suplementación tardía de hierro en niños con peso de nacimiento menor a $1.301 \mathrm{~g}$. Pacientes y Método: Recién nacidos de muy bajo peso (RNMPN) fueron randomizados a recibir suplementación temprana de hierro de $3 \mathrm{mg} / \mathrm{kg} /$ día, tan pronto estaban tolerando alimentación

Recibido el 8 de mayo de 2012, devuelto para corregir el 4 de julio de 2012, segunda versión 23 de agosto de 2012, tercera versión 13 de marzo de 2013, aceptado para publicación el 01 de abril de 2013.

Laboratorio Pasteur proporciono Hierro (Ferrigot gotas) y el placebo, pero no tuvo ninguna intervención en el estudio.

Este trabajo cumple con los requisitos sobre consentimiento /asentimiento informado, comité de ética, financiamiento, estudios animales y sobre la ausencia de conflictos de intereses según corresponda. 
enteral de $100 \mathrm{ml} / \mathrm{kg} /$ día, o a los 61 días de vida como suplementación tardía. Se midió niveles de hemoglobina al inicio de la suplementación temprana de hierro y a los 2 meses de vida. La transfusión de glóbulos rojos fue restringida de acuerdo a las guías de transfusión y no se administró eritropoyetina. Resultados: No hay diferencias en el número de transfusiones de glóbulos rojos entre los grupos y en relación a las morbilidades asociadas con la prematuridad no habría diferencias significativas. Conclusiones: La suplementación temprana de hierro cuando el niño este tolerando $100 \mathrm{ml} / \mathrm{kg} /$ día de leche, no disminuiría la incidencia de las transfusiones de glóbulos rojos en relación al inicio tardío de hierro a los 61 días de vida y probablemente sea segura en los niños menores de $1.301 \mathrm{~g}$.

(Palabras clave: Anemia, prematurez, sulfato ferroso, transfusión, glóbulos rojos).

Rev Chil Pediatr 2013; 84 (4): 379-386

\section{Introducción}

El aumento de la sobrevida de los recién nacidos de muy bajo peso en el último tiempo ${ }^{1}$ ha significado un reto para el equipo médico en términos de llegar a cubrir todas sus necesidades específicas con el objetivo no sólo de mejorar la sobrevida, crecimiento y desarrollo neurológico, sino también su calidad de vida a futuro.

Los recién nacidos menores de $1.500 \mathrm{~g}$ dada su prematurez deben enfrentar múltiples complicaciones, si bien se han dado avances importantes como es el caso de enfermedad de membrana hialina y surfactante entre otras, quedando aún problemas sin resolver, como es el caso de la anemia de la prematurez, que se caracteriza por un descenso de hemoglobina, reticulocitopenia, hipoplasia de médula ósea y concentraciones de eritropoyetina endógena que son bajas en relación con el grado de anemia $^{2-4}$. Una de las causas más comunes de ésta anemia no fisiológica es la pérdida de sangre iatrogénica como resultado de frecuentes tomas de muestras 5 .

Para prevenir la anemia y el riesgo asociado a una transfusión de glóbulos rojos hay algunas estrategias que incluyen:

Retrasar el pinzamiento del cordón umbilical por 30-120 s reduce la transfusión de glóbulos rojos en relación al pinzamiento temprano RR: 2,01 $(1,24-3,27)^{6}$.

Optimizar toma de muestras de sangre, limita la severidad de anemia ${ }^{7}$, además el desarrollo de microtécnicas de laboratorio y de métodos de monitorización no invasivos han permitido reducir la pérdida de sangre ${ }^{8}$.

En relación a la Eritropoyetina recombi- nante como estimulador de eritropoyesis, varios estudios concluyen que guías estrictas de transfusión de glóbulos rojos puede tener el mismo efecto que la administración de eritropoyetina en niños prematuros ${ }^{9}$.

La suplementación de vitamina E para minimizar la severidad de la anemia a dosis de 25 IU puede prevenir anemia hemolítica en el prematuro $^{10}$.

En relación al hierro, los prematuros son más susceptibles a la deficiencia por su baja reserva y su rápido crecimiento en los primeros meses de vida. Hay varios exámenes de laboratorio disponibles para evaluar esta deficiencia, como son el nivel de hemoglobina $(\mathrm{Hb})$, y los niveles de ferritina sérica, que tiene una sensibilidad de $86 \%$ y una especificidad de $92 \%$ en pesquisar deficiencia de hierro con valores menores de $12 \mathrm{ug} / 1^{11}$, teniendo claro que el patrón de referencia de reserva de hierro es la médula ósea. Sin embargo, poco se conoce en relación al tiempo y la dosis de suplementación de hierro en niños de esta edad, no habiéndose reportado efectos adversos con la administración temprana de hierro ${ }^{12}$, ya que teóricamente por medio del estrés oxidativo podría incrementar la displasia broncopulmonar (DBP), hemorragia intraventricular (HIV), retinopatía de la prematuridad (ROP) y enterocolitis necrotizante (NEC) ${ }^{13-16}$.

El año 2010 la Academia Americana de Pediatría y su Comité de Nutrición recomendaron suplementar con hierro a todos los recién nacidos menores de 37 semanas de gestación (SG) iniciando al mes de vida y continuando hasta los 12 meses a dosis de $2 \mathrm{mg} / \mathrm{kg}^{17}$.

Al revisar la literatura hay tres estudios que evalúan el inicio temprano de hierro versus 
tardío. El primero de ellos tenía como objetivo secundario evaluar la necesidad de transfusiones de glóbulos rojos, observando una tendencia a reducir la necesidad de transfusiones en un $33 \%$ cuando se administraba el hierro en forma temprana ${ }^{18}$. El segundo estudio fue realizado en niños de mayor edad gestacional y que recibían leche materna exclusiva, demostrando un efecto significativo en la necesidad de transfusiones (p: 0,045) ${ }^{19}$, y el tercer estudio no mostró efecto en la necesidad de transfusiones en relación a la administración de hierro temprano versus tardío ${ }^{20}$.

Considerando que los prematuros menores de 30 semanas de gestación tienen más riesgo de ser transfundidos, en promedio 7,8 transfusiones durante su estadía hospitalaria ${ }^{21}$, planteamos que sería importante conocer el efecto de la suplementación de hierro precoz en la disminución de los requerimientos y número de transfusiones, y la incidencia de anemia sin incrementar los efectos colaterales versus la suplementación tardía.

\section{Objetivo principal}

Determinar el número y volumen de glóbulos rojos transfundido en relación a la suplementación de hierro precoz versus tardío.

\section{Objetivos secundarios}

Determinar el efecto de la suplementación precoz de hierro versus tardío en relación con la incidencia de ROP, NEC, DBP, HIV.

Determinar la incidencia de anemia en relación con la suplementación temprana de hierro versus tardío, evaluada ésta como niveles de $\mathrm{Hb}$. en sangre total.

\section{Pacientes y Método}

\section{Población de estudio}

Estudio clínico, aleatorizado y prospectivo realizado en la Unidad de Neonatología del Hospital Luis Tisné B. de Santiago de Chile. Se incluyó todos los niños con peso de nacimiento menor de $1.301 \mathrm{~g}$ ingresados entre junio de 2006 y julio de 2007, y que no presentaron malformaciones mayores, enfermedad hemolítica o síndrome de transfusión feto fetal. Previo al ingreso al estudio se obtuvo un consentimiento informado de los padres y fue aprobado por el Comité de Ética del hospital.

\section{Aleatorización}

La aleatorización se realizó a través de un programa computacional que generó números al azar, usando la técnica de bloques de 4, estratificando por peso de nacimiento en menores o igual a $800 \mathrm{~g}$ y en mayores a $800 \mathrm{~g}$ y si recibieron o no transfusiones de glóbulos rojos previo al ingreso al estudio para limitar la posibilidad de desbalances en el tratamiento en estudio en estos grupos con riesgo diferente. El enmascaramiento de la aleatorización se hizo a nivel de farmacia donde no conocían los pacientes que ingresarían al estudio. La asignación a los grupos de estudio se realizó cuando los niños estaban tolerando $100 \mathrm{ml} / \mathrm{kg} /$ día de leche.

\section{Grupos de tratamiento}

En el grupo tratamiento la suplementación temprana de sulfato ferroso $\left(\right.$ Ferrigot $^{\circledR}$ laboratorios Pasteur) se inició a dosis de $3 \mathrm{mg} / \mathrm{kg} /$ día cuando el niño estuvo tolerando $100 \mathrm{ml} / \mathrm{kg} /$ día de alimentación enteral, mientras al grupo control se administró un placebo que tenía aspecto físico idéntico al medicamento del grupo tratado al igual que su envase, por lo que pacientes y clínicos estaban ciegos a la intervención.

La alimentación en ambos grupos fue con leche materna fortificada con fortificante de leche humana (FLH) al 4\% con el fin de alcanzar un buen aporte proteico y calórico de 3,5-4 g/ $\mathrm{kg} /$ día y $130 \mathrm{cal} / \mathrm{kg} /$ día respectivamente, o fórmula de prematuro Similac Special Care, que tiene un aporte proteico y calórico de 2,2 g/100 $\mathrm{ml}$ y de $81 \mathrm{cal} / 100 \mathrm{ml}$ de leche y alto contenido de hierro 1,5 mg/100 $\mathrm{ml}$ de leche. Del volumen total de leche que recibían los $\mathrm{RN}$, el 30-40\% correspondía a leche materna y el resto a fórmula. En algunos casos con sospecha de NEC se suspendió en forma transitoria el aporte de hierro y/o placebo para posteriormente volver a reiniciar cuando el paciente era estabilizado.

Se realizó pinzamiento tardío del cordón dependiendo la condición clínica del recién nacido, y no se administró vitamina $\mathrm{E}$ a dosis farmacológica. 
No se administró eritropoyetina y las transfusiones de glóbulos rojos fueron indicadas por el médico tratante quien era ciego al estudio, de acuerdo a guías estrictas de transfusión ${ }^{22}$ con un volumen a transfundir de $15 \mathrm{ml} / \mathrm{kg}$.

\section{Guías de transfusión de glóbulos rojos}

a. RN con hematocrito (Hto) igual o inferior a $35 \%$ ( $\mathrm{Hb}$ igual o menor a $11 \mathrm{~g} / \mathrm{dl})$ y que esté en ventilación mecánica (VM) con $\mathrm{FiO}_{2}$ sobre 0,40 , DAP sintomático, DBP, cardiopatía congénita cianótica, septicemia con compromiso hemodinámico.

b. RN con Hto igual o menor de $30 \%$ (Hb menor o igual a $10 \mathrm{~g} / \mathrm{dl}$ que esté con VM mínima o CPAP $>6 \mathrm{~cm} \mathrm{H}_{2} \mathrm{O}$ y $\mathrm{FiO}_{2}$ menor al 0.40

c. $\mathrm{RN}$ con Hto igual o menor a $25 \%$ (Hb menor a $8 \mathrm{~g} / \mathrm{dl}$ ) si recibe $\mathrm{O}_{2}$ suplementario o está en CPAP y aumenta sus requerimiento de $\mathrm{O}_{2}$ o de PEEP en las últimas $24 \mathrm{~h}$, apneas a repetición, taquicardia sobre $180 \mathrm{x}$ min o FR sobre $80 \mathrm{x}$ min por más de $24 \mathrm{~h}$, intervención quirúrgica mayor, ganancia de peso inferior a $10 \mathrm{~g}$ por día.

d. RN asintomático con Hto menor o igual a $20 \%$ ( $\mathrm{Hb}$ menor de $7 \mathrm{~g} / \mathrm{dl})$ y recuento de reticulocitos menor de $3 \%$.

La medicación de todos los integrantes del estudio fue reemplazada por $3 \mathrm{mg} / \mathrm{kg} /$ día de sulfato ferroso a los 61 días de edad cronológica y la indicación fue continuar durante el primer año de vida.

\section{Medidas de resultado}

En relación al objetivo principal sobre el número y volumen de transfusiones de glóbulos rojos, estas se evaluaron a los 60 días de vida o al alta.

Se midió niveles de $\mathrm{Hb}$ a las 2 semanas y a los 2 meses de vida utilizando un contador hematológico de marca Sysmex XS 1000i.

En relación con la posible incidencia que tendría el hierro sobre la ROP, DBP, NEC, HIV. El examen oftalmológico se realizó a las cuatro semanas de vida por oftalmólogo, quien no conocía a qué grupo fue asignado cada paciente. Los hallazgos del fondo de ojo fueron registrados de acuerdo a la clasificación internacional de $\mathrm{ROP}^{13}$. DBP fue considerada a la dependencia de 02 mayor a $21 \%$ para saturar más de $92 \%$ a las 36 semanas de edad gestacional corregida y hallazgos radiológicos característicos $^{14}$ que fue interpretada por médico ciego al estudio. NEC se diagnosticó de acuerdo a la clasificación de Bell's ${ }^{15}$, y HIV fue clasificada de grado I a IV según el sistema de Papile ${ }^{16}$ mediante examen que se realizó por médico ciego a la intervención, para lo cual se controló con eco encefálica en las primeras 72 h y luego según su evolución.

\section{Organización}

La persona encargada de monitorizar el estudio, asignar resultados y coleccionar los datos estuvo a cargo del investigador principal quien fue ciego a la intervención.

\section{Tamaño muestral}

Considerando que en el hospital Luis Tisne B. el $80 \%$ de los prematuros menores de 1.301 son transfundidos durante su estadía hospitalaria, nosotros pretendimos detectar una disminución de un 30\% en las transfusiones de glóbulos rojos al administrar el hierro en forma precoz, para lo cual calculamos una población total de estudio de 70 niños, asumiendo un error alfa de 0,05 y un error beta de $20 \%$.

\section{Estadística}

Quien realizó el estudio estadístico si conocía la intervención asignada a cada paciente. Se compararon las variables dicotómicas con riesgo relativo e intervalo de confianza del 95\% y las variables continuas con Anova si son de distribución normal o Mann-Whitney/ Wilcoxon Two-Sample Test si no tienen distribución normal ambas con $\mathrm{p}<0,05$.

\section{Resultados}

De junio de 2006 a julio de 2007 nacieron 8.494 niños en el hospital Luis Tisne B. De éstos 125 fueron menores de $1.301 \mathrm{~g}$, y de ellos 70 fueron aleatorizados (figura 1), y 55 niños no ingresaron al estudio ya sea porque fallecieron antes de la aleatorización o tenían malformaciones mayores o sus padres no auto- 


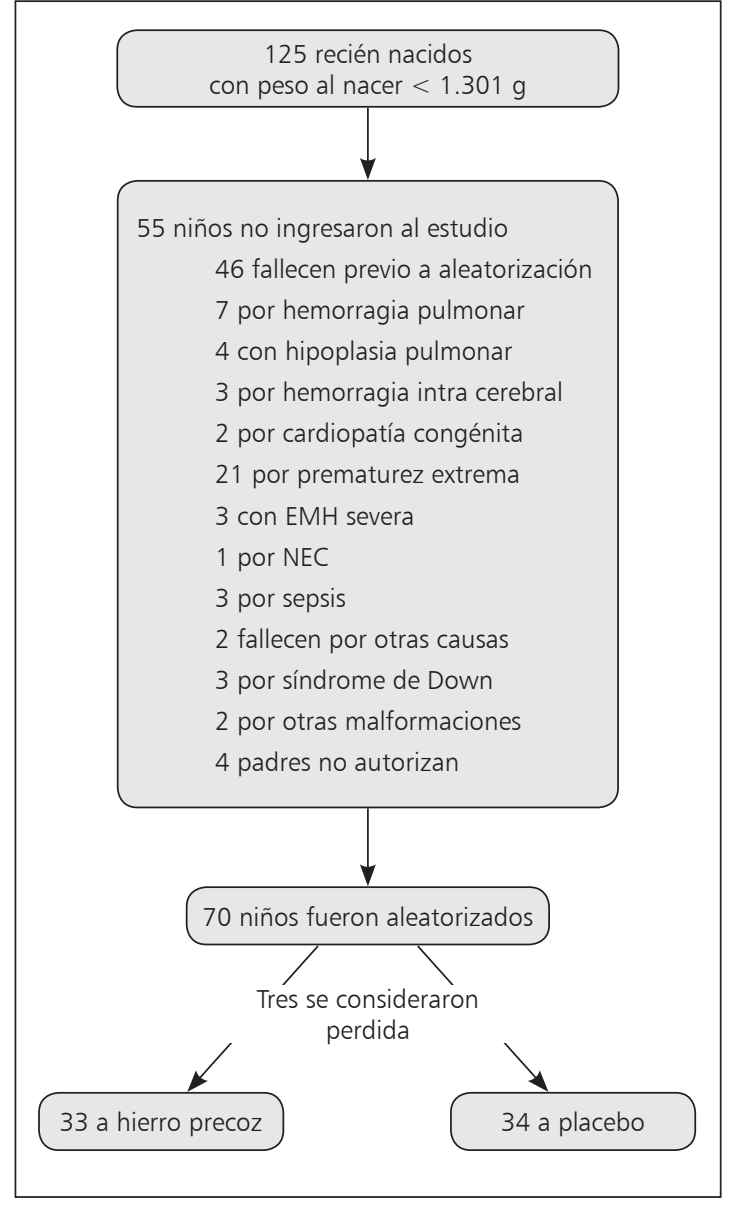

Figura 1. rizaron. Hubieron tres pérdidas, 2 en el grupo de estudio y uno en el grupo control que fueron trasladados a su hospital de origen y no se obtuvo acceso a los resultados finales.

Las características demográficas maternas (tabla 1) son similares entre los grupos así como las características del RN (tabla 2). El $13 \%$ de todas las transfusiones no respetó las guías de transfusión, lo cual fue similar en ambos grupos.

En relación al objetivo principal, ya sea en volumen como en el número de transfusiones de glóbulos rojos no se observó diferencia significativa a los 60 días de vida o al alta, tanto en el grupo de estudio como en el de placebo (tabla 3).

En relación a las morbilidades asociadas con la prematuridad hay similar incidencia entre los grupos (tabla 4).

Los niveles de hemoglobina tanto al inicio como al final del estudio tampoco mostraron diferencia significativa (tabla 5).

\section{Discusión}

De los 70 pacientes que ingresaron al estudio hubo sólo 3 pérdidas, y los resultados obtenidos nos muestran que la administración de hierro precoz a dosis de $3 \mathrm{mg} / \mathrm{kg} /$ día en relación a la administración tardía no disminu-

Tabla 1. Características principales de la madre

\begin{tabular}{|lrc|}
\hline Características & Hierro $\mathbf{n}=\mathbf{3 3}(\mathbf{\%})$ & Placebo $\mathbf{n}=\mathbf{3 4}(\mathbf{\%})$ \\
\hline Edad madre* & $27,0 \pm 8,2$ & $28,7 \pm 6,9$ \\
\hline Primigesta & $8(24,2)$ & $7(20,6)$ \\
Control de embarazo & $28(84,8)$ & $33(97,1)$ \\
Patología del embarazo & & $0(0,0)$ \\
$\quad$ Diabetes mellitus & $1(3,0)$ & $0(0,0)$ \\
Diabetes gestacional & $1(3,0)$ & $9(26,5)$ \\
Pre-eclampsia & $8(24,2)$ & $1(2,9)$ \\
Eclampsia & $3(9,1)$ & $1(2,9)$ \\
Desp. placenta & $1(3,0)$ & $0(0,0)$ \\
Corioamnionitis & $0(0,0)$ & $3(8,8)$ \\
RPO $>18$ h & $3(9,1)$ & $30(88,2)$ \\
Corticoides & $26(78,8)$ & $22 / 30(73,3)$ \\
\hline Profilaxis completa & $20 / 26(76,9)$ & $26(76,5)$ \\
\hline
\end{tabular}

*Promedio y desviación estándar. No se encontró diferencias significativas entre los grupos. 
Tabla 2. Características principales del recién nacido

\begin{tabular}{|lcc|}
\hline Características & Hierro $\mathbf{n}=\mathbf{3 3}(\mathbf{\%})$ & Placebo $\mathbf{~}=\mathbf{3 4}(\mathbf{\%})$ \\
\hline Edad gestacional $(\mathrm{sem})^{*}$ & $27,6 \pm 1,8$ & $28,0 \pm 2,6$ \\
Peso $(\mathrm{g})^{*}$ & $960 \pm 188$ & $959 \pm 212$ \\
Talla $(\mathrm{cm})^{*}$ & $34,7 \pm 2,9$ & $35,2 \pm 2,8$ \\
Hombres & $16(48,5)$ & $16(47,1)$ \\
Apgar 1 min $\leq 3$ & $5(15,1)$ & $4(11,8)$ \\
Apgar 5 min $\geq 7$ & $28(84,8)$ & $30(94,2)$ \\
PEG al nacer & $11(33,3)$ & $19(55,9)$ \\
Transfusión $\leq 7^{\circ}$ día & $8(24,2)$ & $7(20,6)$ \\
$\quad$ Vol. Transf $<7^{\circ}$ día $(\mathrm{ml})^{*}$ & $11,5 \pm 1,7$ & $11,9 \pm 2,8$ \\
Inicio del fierro (días)* & $19,1 \pm 10,7$ & $17,5 \pm 8,4$ \\
EMH & $23(69,7)$ & $22(64,7)$ \\
Surfactante en EMH & $20 / 23(87,0)$ & $19 / 22(86,4)$ \\
VM & $20(60,6)$ & $22(64,7)$ \\
VM días** & $5(1-51)$ & $6(1-62)$ \\
CPAP & $26(78,8)$ & $26(76,5)$ \\
CPAP días** & $5,5(1-24)$ & $4,5(1-24)$ \\
Infección connatal*** & $17(51,5)$ & $14(41,2)$ \\
\hline
\end{tabular}

*Promedio y desviación estándar. **Mediana (mínimo-máximo). ***Ninguna con hemocultivo $(+)$. No se encontró diferencias significativas entre los grupos. PEG: pequeño para edad gestacional. EMH: enfermedad de membrana hialina. VM: ventilación mecánica. CPAP: presión positiva continua de vía aérea.

Tabla 3. Número y volumen de transfusiones de glóbulos rojos en los grupos de estudio

\begin{tabular}{|lccc|}
\hline Resultado & Hierro $\mathbf{n}=\mathbf{3 3}(\mathbf{\%})$ & Placebo $\mathbf{n}=\mathbf{3 4}(\mathbf{\%})$ & RR (IC 95\%) o p* \\
\hline Transfusión $>7$ días & $22(66,7)$ & $23(67,6)$ & $0,97(0,49-1,93)$ \\
\hline$N^{\circ}$ transfusión > 7 días** & $2,9 \pm 1,8$ & $3,0 \pm 2,3$ & 0,83 \\
Vol. transfundido** & $53,6 \pm 25,8$ & $54,2 \pm 33,6$ & 0,95 \\
$N^{\circ}$ transfusión > 7 días*** & $3,7 \pm 2,1$ & $3,6 \pm 2,8$ & 0,87 \\
Vol. transfundido*** & $83,2 \pm 43,5$ & $75,1 \pm 56,5$ & 0,59 \\
\hline
\end{tabular}

*ANOVA. **Seguimiento hasta los 60 días de vida o alta. ${ }^{* * *}$ Seguimiento hasta el alta. No se encontró diferencias significativas entre los grupos.

Tabla 4. Incidencia de morbilidades asociadas a la prematurez

\begin{tabular}{|lccc|}
\hline Morbilidad & Hierro $\mathbf{n}=\mathbf{3 3}(\mathbf{\%})$ & Placebo $\mathbf{n}=\mathbf{3 4}(\mathbf{\%})$ & RR (IC 95\%) \\
DBP (02 a las 36 EGC) & $18(54,5)$ & $14(41,2)$ & $1,29(0,81-2,06)$ \\
Infección intrahospitalaria & $14(42,4)$ & $14(41,2)$ & $1,02(0,68-1,50)$ \\
Retinopatía & $4(12,1)$ & $6(18,2)$ & $0,93(0,76-1,14)$ \\
HIV & $11(33,3)$ & $8(23,5)$ & $1,15(0,85-1,56)$ \\
NEC & $3(9,4)$ & $1(2,9)$ & $1,07(0,94-1,21)$ \\
\hline
\end{tabular}

No se encontró diferencias significativas entre los grupos. DBP: displasia broncopulmonar. HIV: hemorragia intraventricular. NEC: enterocolitis necrotizante.

Tabla 5. Niveles de hemoglobina al inicio y al final del estudio

\begin{tabular}{|lccc|}
\hline Parámetro & Hierro $\mathbf{n}=\mathbf{3 3}$ & Placebo $\mathbf{n}=\mathbf{3 4}$ & $\mathbf{p}^{*}$ \\
\hline Hb inicial & $11,9 \pm 2,5$ & $11,5 \pm 2,5$ & 0,57 \\
\hline Hto inicial & $34,7 \pm 7,9$ & $33,7 \pm 7,5$ & 0,60 \\
Hb final & $10,6 \pm 3,3$ & $11,7 \pm 5,2$ & 0,37 \\
Hto final & $27,7 \pm 4,5$ & $28,0 \pm 7,9$ & 0,84 \\
\hline
\end{tabular}

*Mann-Whitney/Wilcoxon Two-Sample Test. No se encontró diferencias significativas entre los grupos. Hb: hemoglobina. Nto: hematocrito. 
yó el número ni el volumen de transfusión de glóbulos rojos. Debemos destacar que pese a que alcanzamos el tamaño muestral propuesto, la incidencia de transfusión fue más baja que la histórica y los intervalos de confianza de nuestros resultados son amplios, por lo que la aseveración de que sirve o no aún permanece en la duda.

Hay estudios publicados que muestran que la administración temprana de hierro disminuye la frecuencia de transfusiones de glóbulos rojos, uno de ellos se realizó en niños menores de 32 semanas de gestación, en donde se comparó la suplementación temprana de hierro a las 2 semanas de vida dosis de $3 \mathrm{mg} /$ $\mathrm{kg} /$ día en relación a la administración a las 4 semanas y se encontró una disminución significativa en las transfusiones de glóbulos rojos $(\mathrm{p}=0,045)$, siendo el número necesario a tratar para prevenir una transfusión de glóbulos rojos de cinco $^{20}$, pero a diferencia de nuestro estudio tenían un peso promedio de nacimiento mayor al nuestro de $1.200 \mathrm{~g}$ versus $980 \mathrm{~g}$ y se realizó en niños que recibían leche materna exclusiva. En relación al volumen total de alimentación, sólo el 30-40\% fue con leche materna y sólo el $25 \%$ del total de los pacientes del estudio fue con leche materna exclusiva, el resto fue con fórmula de prematuro con mayor contenido de hierro, lo que podría explicar el nulo efecto del hierro precoz en nuestro estudio. Probablemente el hierro precoz tenga mayor beneficio en niños que reciben más leche materna que fórmula de prematuros.

Existen dos estudios que evalúan el inicio temprano de hierro versus tardío con el fin de ver la deficiencia de hierro, el primero de ellos consideró como inicio temprano el aporte de hierro cuando el niño está recibiendo $100 \mathrm{ml} /$ $\mathrm{kg} /$ día de leche y tardío a los 61 días de vida. La población de estudio fue niños menores de $1.301 \mathrm{~g}$ con un peso promedio de nacimiento de $870 \mathrm{~g}$, los que recibieron leche materna y/o fórmula de prematuro características muy similares a nuestro estudio. Los resultados finales mostraron una disminución de la deficiencia de hierro en el grupo precoz y además como salida secundaria se ve una tendencia a reducir la necesidad de transfusiones en un $33 \%$ cuando se administraba el hierro en for- ma temprana, no evidenciando efectos colaterales ${ }^{18}$; el segundo estudio también evaluó deficiencia de hierro y como salida secundaria los requerimientos de transfusiones durante la hospitalización, y concluyeron que no hubo diferencia entre los grupos ${ }^{20}$. Sin embargo, es importante considerar que estos estudios no fueron diseñados para ver la relación de hierro precoz y transfusión de glóbulos rojos por lo que no se podrían sacar conclusiones.

Además se menciona en la bibliografía que la presencia o el exceso de hierro en período perinatal puede producir alteraciones en el desarrollo de algunos órganos ya que los prematuros tendrían un sistema antioxidante inmaduro y son particularmente vulnerables, pero como podemos ver en estudios previos y en nuestro estudio la incidencia de DBP, NEC, ROP, HIV fue similar en ambos grupos por lo que podemos decir que la administración temprana de hierro no se asociaría con eventos adversos.

\section{Conclusión}

La suplementación temprana de hierro de $3 \mathrm{mg} / \mathrm{kg} / \mathrm{día}$, cuando el niño este recibiendo $100 \mathrm{ml} / \mathrm{kg} /$ día de leche, no disminuiría la incidencia del número ni volumen de transfusiones de glóbulos rojos en relación al inicio tardío de hierro a los 61 días de vida y en relación a las morbilidades asociadas a la prematuridad el hierro precoz no incrementaría eventos adversos.

Esta falta de efecto no la podemos confirmar ni descartar, por el amplio intervalo de confianza, por lo que a futuro se requiere un estudio con más pacientes; además nuestros prematuros recibieron más fórmula enriquecida de hierro, a diferencia de la leche materna que su aporte de hierro es menor, por lo que sería importante realizar un estudio en niños que son alimentados con leche materna exclusiva en donde probablemente la administración de hierro precoz sea beneficioso.

\section{Referencias}

1.- Lemons JA, Bauer CHR, Oh W, et al: Very low birth weight outcomes of the National Institutes of Health 
and Human Developmet Neonatal. Pediatrics 2001; 107 : $1-8$.

2.- Doyle JJ: The rol of erythropoietin in the anemia of prematurity. Seminar Perinatol 1997; 21: 20-7.

3.- Wandstrat TL, Kaplan B: Use of erythropoietin in premature infants: Controversies and the future. Ann Pharmacother 1995; 29: 166-73.

4.- Strauss $R G$ : Erytropoietin in the pathogenesis and treatment of neonatal anemia. Transfusion 1995; 35: 68-73.

5.- John A: Vidness. Pathophysiology, Diagnosis, and prevention of Neonatal Anemia. NeoReviews 2000; 1 (4): 61-5.

6.- Rabe H, Reynolds G, Díaz-Rosello J: Early versus delayed umbilical cord clamping in preter. Cochrane database Sist Rev 2004; 18 (4): CD003248.

7.- Ringer SA, Richardson DK, Sacher RA, Keszler M, Hallowell Churchill W: Variations in transfusions practices in neonatal intensive care. Pediatrics 1998; 101: 194-200.

8.- Jeter EK, Spivey MA: Red blood cell transfusions for selected neonatal and pediatric patients, In: Reid ME, Nance SJ. Red Cell Transfusion: A practical guide. Totowa: Humana Press Inc 1998; 89-104.

9.- Franz AR, Pohlandt F: Red blood cell transfusions in very and extremely low birth weight infants under restrictive transfusion guidelines. Is exogenous erythropoietin necessary? Arch Dis Child Fetal Neonatal 2001; 84: 96-100.

10.- American Academy of Pediatrics Committee on $\mathrm{Nu}$ trition: Nutritional needs of low birth weight infants. Pediatrics 1985; 75: 976-80.

11.- Reibo E: Diagnosis of iron deficiency. Scand J Hematol 1985; 43 (Suppl): 5-39.

12.- Van Zoeren Grobben D, Moison RMW: Iron containing feeding formula and oxidative stress in preterm babies. Pediatr Res 1998; 43: 270.

13.- International Committee for the Classification of Retino- pathy of Prematurity: The Intenational Classification of Retinopathy of Prematurity revisited. Arch Ophthalmol 2005; 7: 991-9.

14.- Bancalari E, Claure N, Sonsenko IRS: Bronchopulmonary Dysplasia:changes in pathogenesis epidemilogy and definition. Seminars in neonatology 2003; 8: 6371.

15.- Kliegman RM, Hack M, Jones P, Fanaroff AA: Epidemiologic study of necrotizing enterocolitis among low-birth-weinght infants. Absence of identifiable risk factores. J Pediatr 1982; 100 (3): 440-4.

16.- Papile LA, Burstein J, Burstein R, Koffer H: Incidence and evolution of subependymal and intraventricular hemorrhage: a study of infants with birth weinghts less than 1,500 gm. J Pediatr 1978; 92 (4): 529-34.

17.- Baker RD, Greer FR; Committee on Nutrition American Academy of Pediatrics: Diagnosis and prevention of iron deficiency and iron-deficiency anemia in infants and young children (0-3 years of age). Pediatrics 2010; 126 (5): 1040-50.

18.- Franz AR, Mihatsch WA, Sander S, Kron M, Pohlandt $F$ : Prospective Randomized Trial of Early Versus Late Enteral Iron Suppementation in infants with a birth weight of less than 1301 grams. Pediatrics 2000; 106: 700-6.

19.- Arnon Shmuel, Shiff Yakov, Litmanovitz Ita, et al: The Efficacy and Safety of Early Supplementation of Iron Polymaltose Complex in Preterm Infants. American Journal of Perinatology 2007; 24 (2): 95-9.

20.- Jeeve SM, Saxena R, Mani K, Agarwal R, Deorari AK, Vinod $K P$ : Early iron supplementation in very low birth weight infant a randomized controlled trial. Acta Paediatrica 2009: 98; 953-8.

21.- Schwarz KB, Dear Pr, Gill AB, Newell SJ, Richards M: Effects of transfusion in anemia of prematurity. Pediatr Hematol-Oncol 2005; 22: 551-9.

22.- Christensen $R$ : Hematologic problems of the neonate, WB Saunders, Philadelphia, 200. 